\title{
Properties of spatial representations: Data from sighted and blind subjects
}

\author{
RALPH NORMAN HABER and LYN R. HABER \\ University of Illinois, Chicago, Illinois \\ CHARLES A. LEVIN \\ Baldwin-Wallace College, Berea, Ohio \\ and \\ REBECCA HOLLYFIELD \\ Hines Veterans Administration Hospital, Hines, Illinois
}

\begin{abstract}
Five questions concerning the properties of spatial representations are explored. (1) How accurately does a spatial representation correspond to the true scene? (2) If inaccurate, how does it differ? (3) Are representations of a familiar scene more accurate than those of an unfamiliar one? (4) Do representations of a scene currently in view differ from those retained in memory? (5) Do the representations of the blind have properties comparable to those of the sighted? Seven sighted and 7 highly mobile blind subjects, all familiar with a room, and 6 sighted subjects unfamiliar with it, were asked to estimate the absolute distances between 10 salient objects in the room. The 14 familiar subjects made their estimates twice: while they were in the room, and while they were remote from it. Regression analyses showed that the estimates of all subjects had strong metric properties, being linearly related to true distance, with a true zero point; and multidimensional scaling showed that all subjects produced distance estimates that could be scaled in two dimensions to closely match the actual locations of the objects. Familiarity had no effect. The effect of location of testing was the same for both the sighted and the blind: all subjects displayed better spatial knowledge when tested in the room; and all subjects underestimated true distance substantially when tested out of the room. The results showed no qualitative differences as a function of blindness, at least for these highly skilled blind travelers.
\end{abstract}

A person's ability to move about in an environment depends on the successful integration of concurrent sensory information and an internal spatial representation of the environment (see Haber, 1985a, 1990, for a detailed review and discussion). Research on sensorily guided locomotion in normally sighted subjects has emphasized the first of these two components but has provided relatively little description or quantification of the second. Even the space science work on the development of guidance systems for autonomous robots has been dis-

We would like to thank the staff of the Blind Center of the Hines VA Hospital for their cooperation in permitting us to use their lobby, and for permission to disrupt their schedules, as well as take up some of their time. Partial support for this project was provided by two research contracts from the Veterans Administration Rehabilitation Research and Development Research Program (C448-R and C995-PA) to the Rehabilitation Research and Development Center at Hines VA Hospital, and through contracts from Hines VA Hospital to the University of Illinois, Chicago. Partial support was also provided by a research grant from the National Eye Institute (EY07801) to the first two authors. We would like to thank Kevin Novak, James Gramata, Deirdre McConathy, Rebecca Burzette, Richard Marcantonio, and Lynn Ostergren for their assistance and for their comments on earlier drafts of this paper. We also benefited from the anonomous comments of several reviewers. Correspondence should be directed to R. N. Haber, Department of Psychology $(\mathrm{m} / \mathrm{c} 285)$, University of Illinois, Chicago, IL 60680. proportionately focused on sensory input, and much less so on how the incoming information might be represented (see Haber \& Haber, 1991, for a critique of robotic navigation). Research on the characteristics of spatial representations, on the other hand, has come primarily from studies of memory in sighted subjects (e.g., Tversky, 1981 ), in which the properties of the sensory processing are unknown or simply assumed to be veridical; and from studies of blind subjects (see, e.g., Foulke, 1985; Haber, 1985b; Rieser, Hill, Talor, Bradfield, \& Rosen, 1992; Warren, 1984), in which the sensory information has again accumulated from the past, and its properties are unknown.

Because of this separation of focus, and a multiplicity of assessment procedures, several questions concerning properties of spatial representations themselves have not been adequately addressed: (1) To what extent does a spatial representation of a scene mirror the actual scene? (2) When not veridical, how does it differ? (3) Are spatial representations of a familiar scene more accurate than those of an unfamiliar one? (4) Do spatial representations of a scene currently in view (constructed directly from visual processes) differ from spatial representations retained in memory of scenes not currently in view? (5) Do spatial representations of the blind have properties comparable to those of normally sighted people? 
We consider each of these questions here.

1. To what extent does a spatial representation of a scene mirror the actual scene? Recent evidence supports the assumption that normally sighted people do have veridical spatial representations, at least when the scene is in view (Haber, 1985a). Using subjects' absolute distance estimations as the assessment method, Toye (1986), Wagner (1985), Przeorek (1986), and Levin and Haber (in press) found strong evidence for map-like representations.

Toye (1986) also compared three measures of spatial cognition: in addition to estimating absolute distances, his subjects drew maps of the locations of the objects in the scene, and they estimated relative distances. These three response measures produced similar representations, each of which closely resembled the actual scene. Toye showed further that the absolute distance estimates were highly correlated with the true distances: the relation was linear, with a slope of unity and an intercept of zero-a ratio scale of distance. This finding was replicated by Levin and Haber (in press).

This pattern of results supports the assumption that sighted people have spatial representations that closely mirror the true scene. Since in the statistical analyses the judgments of the subjects were compared with a true survey map of the scene, even though the subjects in all of these experiments had never viewed the scene from any perspective prior to being tested, and even though they made their judgments exclusively from an eye-level perspective, these results also show that sighted subjects can and do translate ground-level perspectives to construct and represent survey map representations.

2. However, each of these studies with the sighted also showed that there was a small, very similar distortion in the cognitive maps constructed from the absolute distance estimates: perceived space was shrunk along one of its two axes. Detailed multiple regression analyses by Przeorek (1986) and especially by Levin and Haber (in press) showed that the dimension of space perpendicular to the line of sight (i.e., parallel to the horizon) was overestimated, whereas the dimension of space extending from the observer and aligned with the line of sight was represented accurately. Analyses of the distances between specific pairs of objects verified this slight distortion. In addition, Levin and Haber showed that two objects appeared to be farther apart from each other (holding their true separation constant) as a function of the angle subtended by the observer's eye turn and/or head turn made in order to shift the view from one object to the other. This visual angle, or more generally, angle subtended effect produces little distortion in the perception of separation distances between objects that are closely aligned with the line of sight, since a very small turn is all that is needed for one to look from one object to the other. However, it accounts for some overestimation in the perception of the separation distance between pairs of objects that are perpendicular to the line of sight, since much larger turns are needed for one to look from one object to the other.

These findings suggest that the spatial representations of the sighted have the properties of survey maps and are highly accurate, but that they contain systematic distortions that are presumably produced at the time of current viewing of the scene and are induced by asymmetries in the two dimensions of space when one is viewing from a ground-level perspective. Thus, the map in a sighted person's head does not exactly match a survey map of the same scene, even when the person is looking at the scene.

3. Are spatial representations of a familiar scene more accurate than those of an unfamiliar one? As far as we know, scene familiarity has not been systematically manipulated in any of the existing research on spatial representations. In virtually all such research with normally sighted subjects, unfamiliar scenes have been presented to control for the effects of memory, whereas in research on spatial representations in memory, the scenes have primarily been familiar. Research on the blind has relied on highly familiar scenes. Therefore, we know next to nothing about the effects of familiarity on the properties of spatial representations.

4. Do spatial representations of a scene currently in view differ from those retained in memory? It seems plausible that the spatial representation of a scene not in view - that is, a representation reported from memorymight show distortions when compared with a survey map. A substantial research literature (see Tversky, 1981) confirms that peoples' long-term memories of large-scale scenes show systematic distortions: angles, lengths, and curves tend to be regularized, equalized, and straightened, respectively. Similar effects have been shown in spatial representations extracted from short-term memory. For example, Kosslyn, Pick, and Farello (1974) showed that relative distance estimates made by children could be scaled to resemble the scene they had previously studied, but with significant inaccuracies in the representations that systematically reflected properties of the structure of the scene.

Unfortunately, there are no data in which the same subjects have been assessed both when looking at a scene and when reporting it from memory. Therefore, it has not been possible to tease apart distortions arising in perception itself from those contributed by the memorial process.

5. Do spatial representations of the blind have properties comparable to those of the sighted? This question dates back to an important philosophical tradition of the 18th century, in which Molyneux, Locke, and Berkeley argued the following question: Is vision necessary for the acquisition of principles of spatial organization? Presently, the commonly accepted notion is that blind people do not understand spatial layout in the same manner as do the sighted. This acceptance has been bolstered by a substantial research literature extending from the 1950 s (as well as centuries of previous speculation), showing that blind people have less accurate spatial representations than sighted people do (see Warren, 1984, for a summary; cf. Haber \& Haber, 1993, for a critique).

There have been a number of theoretical discussions of spatial representations in blind persons (e.g., Heller \& Kennedy, 1990; Landau, 1988), and of how they might differ from those of the sighted. One of the most important theories has been adapted from Siegel and White 
(1975), who originally considered the acquisition of spatial knowledge in children.

Siegel and White (1975) proposed a three-way qualitative distinction among levels of spatial representations. The highest level of spatial representation has a surveymap-like global character, such that all spatial relationships among the objects in a scene are represented equally accurately. This level is assumed for normally sighted adults, an assumption supported by the kind of research briefly reviewed above. It is also assumed for blind people with high levels of spatial and travel skills, though with much less evidence: the typical assumption is that few blind people have this kind of spatial representation. At the second level, the representation is limited to accurate knowledge of the locations of objects along the routes traveled by the subject. Some blind travelers are assumed to acquire this level. At the lowest level, the representation includes knowledge only of the location and identification of a few "landmark" objects, a level that many blind persons are assumed never to surpass.

In a number of studies, these three levels have been used to describe data from blind subjects. For example, Hollyfield and Foulke (1983) had blind and sighted subjects travel a new route several times and, after each trip, construct from memory a model of the route containing the landmarks and other objects encountered. The blind subjects showed adequate evidence of a route-based representation (though they had inadequate representations of curves), but they were poorer than sighted subjects in survey-map-like representations that included nonroute relations among the objects. Rieser, Lockman, and Pick (1980) asked blind and sighted subjects to make relative distance estimations among objects in a highly familiar indoor scene. The estimates of the blind subjects were sufficiently accurate to be scaled with multidimensional scaling (MDS) to resemble the scene. However, this was the case primarily when the judgments reflected routes actually traveled rather than straight-line distances between the objects, again suggesting more route knowledge than map-like knowledge. This limitation was especially pronounced for the early blind subjects. Lockman, Rieser, and Pick (1981) reported similar results for a familiar large urban scene and also showed that the quality of the spatial representations of the scene was related to the mobility skill of the blind subjects. Brambring (1985), using primarily verbal reports about familiar routes, was able to construct evidence for at least route representations of the spaces. Finally, Rieser, Guth, and Hill (1986) asked blind (and sighted) subjects to point to the locations of objects in an indoor space. They found that all subjects could accurately point to an object after having walked to the object that preceded it on a route, but when they had to do it from memory, the early blind subjects had much greater difficulty than the sighted and late blind subjects had.

In these studies, a variety of methods were used to assess spatial representations, including model reconstruc- tions, relative distance estimates, verbal reports, and pointing responses in blind travelers. The results constitute most of the evidence used to support the conclusion that although blind persons have spatial representations of the scenes in which they move, those representations lack some of the global survey-map-like knowledge of the complete layout of the scene available to the sighted. Early and late blind subjects are also found to differ from one another, with the former displaying more impoverished or inaccurate representations.

The purpose of the present experiment was to provide some comparative data on the properties and distortions of spatial representations in the sighted and the blind, with (1) assessment method, (2) familiarity, and (3) viewing conditions controlled.

First, to control for the effects of assessment method, the same method was used for all subjects in all conditions, one that has been used extensively with the sighted, and that has proved to be powerful. All subjects were asked to estimate the absolute distances between all possible pairs of a group of preselected objects in the scene. Although no previous experiment had required blind subjects to make absolute distance estimates, pilot testing suggested that at least experienced blind travelers could make such estimates adequately. Since absolute estimates lend themselves to more powerful analyses than relative estimates do, this experiment elicited only the former. ${ }^{1}$

Absolute distance estimates have been analyzed in the literature in two ways in order to assess spatial representations in the sighted. In the first, the estimates from each subject separately are submitted to MDS to examine the degree to which the true coordinates of the object locations in the actual scene match the locations constructed from the scaling of the estimates. This statistical procedure has been successfully applied to data from the blind as well, though on the basis of relative distance estimates. In the second procedure, the estimated distances are used in multiple regression analyses to determine both the relationships between true and estimated distances, and the relative contributions of different predictors of those estimates. Both procedures were used in the present experiment.

Second, the effects of familiarity are usually confounded in the existing data. In the present experiment, sighted subjects were therefore composed of two groups, one familiar with the experimental scene, and another unfamiliar with it. This permitted both groups to be contrasted with a third group of blind (and necessarily familiar) subjects. For obvious experimental reasons, we could not test the spatial knowledge of blind subjects about a scene unfamiliar to them. Therefore, our conclusions regarding familiarity apply only to sighted subjects.

Third, to assess the differences between concurrent sensory stimulation and memory, the sighted (familiar) subjects as well as the blind (familiar) subjects were asked to make their judgments about the same scene twice: while seated in the scene, and from a remote location, where only their memory of the scene was available to them. 


\section{METHOD}

\section{Subjects}

Twenty employees of the Hines, IL, Veterans Administration Hospital served as subjects. Each was tested individually in two or three 1-h sessions. The subjects were divided into three groups. Each member of the blind-familiar group $(n=7)$ and the sightedfamiliar group $(n=7)$ was associated with the Blind Center of the Hospital and used the Blind Center lobby (the experimental space) extensively as a place to meet and instruct clients, and as a general social gathering area. Finally, each member of the sighted-unfamiliar group $(n=6)$, while employed in the hospital, had never before visited the Blind Center (the hospital's first floor alone extends nearly a mile), so these subjects saw the entry lobby for the first time at the beginning of the experiment. The sexes in all three groups divided roughly evenly. Ages ranged from 22 to 55: the three groups had about the same age range.

Each of the 7 blind subjects was employed at the Blind Center, though none were involved in the orientation and mobility training of clients. Each was a highly experienced traveler and used a long cane regularly. Each either was totally blind, or could distinguish only light from dark: none had any pattern perception. Four of these blind subjects had lost their vision as adults, 2 were congenitally blind, and the last lost his vision before age 6 . There was no correlation between age at time of testing and age of vision loss.

\section{Experimental Space and Objects}

All subjects were tested for their spatial knowledge of the entry lobby of the Blind Center. A map of this space is shown in Figure 1 , and two photographs taken from different positions in the space appear in Figure 2.

Each subject was initially brought into the experimental space and seated at Position $A$ in a chair located against a pillar. The location of the seated subject at Position $A$ was the first object used for testing. Nine additional test objects are labeled B-J on Figure 1. Two criteria were used to select the 10 objects included in the experiment. First, the position and function of the objects were well known and salient to the blind persons who used the room. Second, each of the 9 objects B-J was visible from Position A, where the subject sat (if the subject was sighted). It was not required that all 9 objects be simultaneously visible in the same glance: for example, subjects had to turn their heads substantially to look from the chair near the desk (I) to the bannister (J).

\section{Location of Testing}

The subjects in the blind-familiar and in the sighted-familiar groups were tested on two separate occasions. The first remote testing was carried out in a small office room across the hall from the Blind Center's lobby. Each subject was taken there by the experimenter after the initial visit to the experimental space and the labeling of the test objects. The remote room contained a table and two chairs for the subject and experimenter. The in-room testing was always done while the subject sat at Position A in the experimental space. The instructions and procedures were the same as for the remote testing. Since the sighted-unfamiliar subjects were not familiar with the experimental space, they only had in-room testing. There were no blind-unfamiliar subjects tested at all, since they could not have performed the tasks without having had previous contact with the objects in the lobby.

\section{Instructions and Testing Procedures}

All subjects were tested individually by a single experimenter. The sighted subjects were tested with their normal vision intact; no blindfolds were used. Regardless of group, the subject was brought into the entry lobby and asked to sit in the chair marked A on Figure 1. The study was explained as one concerned with people's sensitivity to their environment and its layout. The blind

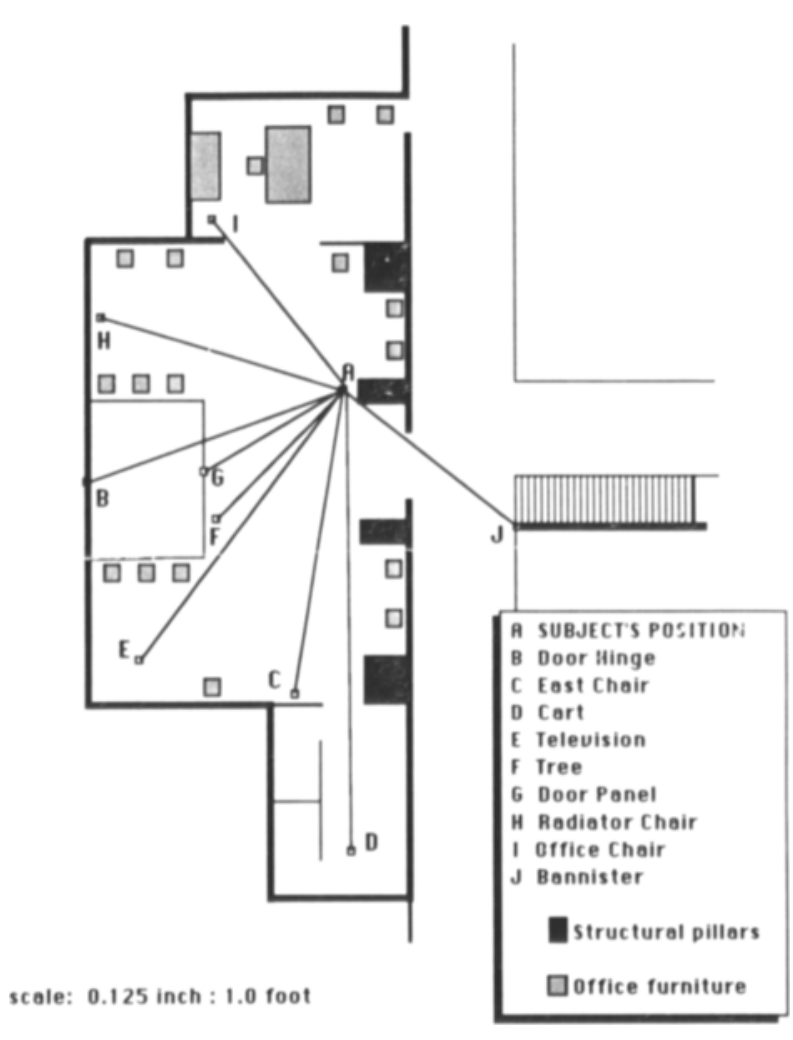

Figure 1. The two-dimensional layout of the experimental room, scaled at $8 \mathrm{ft}=1$ in. The subject sat at Position A (when being tested in the room) and made judgments of the distances between all possible combinations of the 10 locations A-J. The other items of furniture also indicated can be identified with reference to the photographs in Figure 2.

subjects provided basic background information regarding their blindness. Then the 10 objects of relevance were named, including the chair in which the subject was seated. If the subject was in either the blind-familiar or the sighted-familiar group, he/she walked to each object, identified it, and then rated its familiarity twice: the first time on how familiar it was; and the second time on how often he/she came into direct contact with it or came near enough to know that it was within touching distance. The familiarity ratings were recorded on a prepared data sheet by the experimenter. The $10 \mathrm{ob}-$ jects were all sufficiently familiar to each subject that no analyses of the individual familiarity of objects could be attempted: every object was rated as familiar or highly familiar by every subject in the two familiar groups.

For each of the 14 subjects in the two familiar groups, after each of the objects to be used in the testing was labeled for the subject, and the familiarity ratings were completed, the subject was taken across the hall to the remote room. There, the subject was instructed to estimate the distance between every pair of objects using an $a b$ solute distance metric (e.g., feet or meters). Two subjects preferred to use meters and centimeters; the remainder used feet. Each pair of objects was named by the experimenter, and the subject responded verbally with an estimate of the straight-line distance separating them. The subject was instructed not to round off to multiples of 5 or $10 \mathrm{ft}$, but to make estimates to the nearest half foot (or fraction of a meter). After the subject gave a response, the experimenter recorded it on a prepared data sheet. The experimenter selected the first pair randomly from the ordered list of 45 object pairs and then 

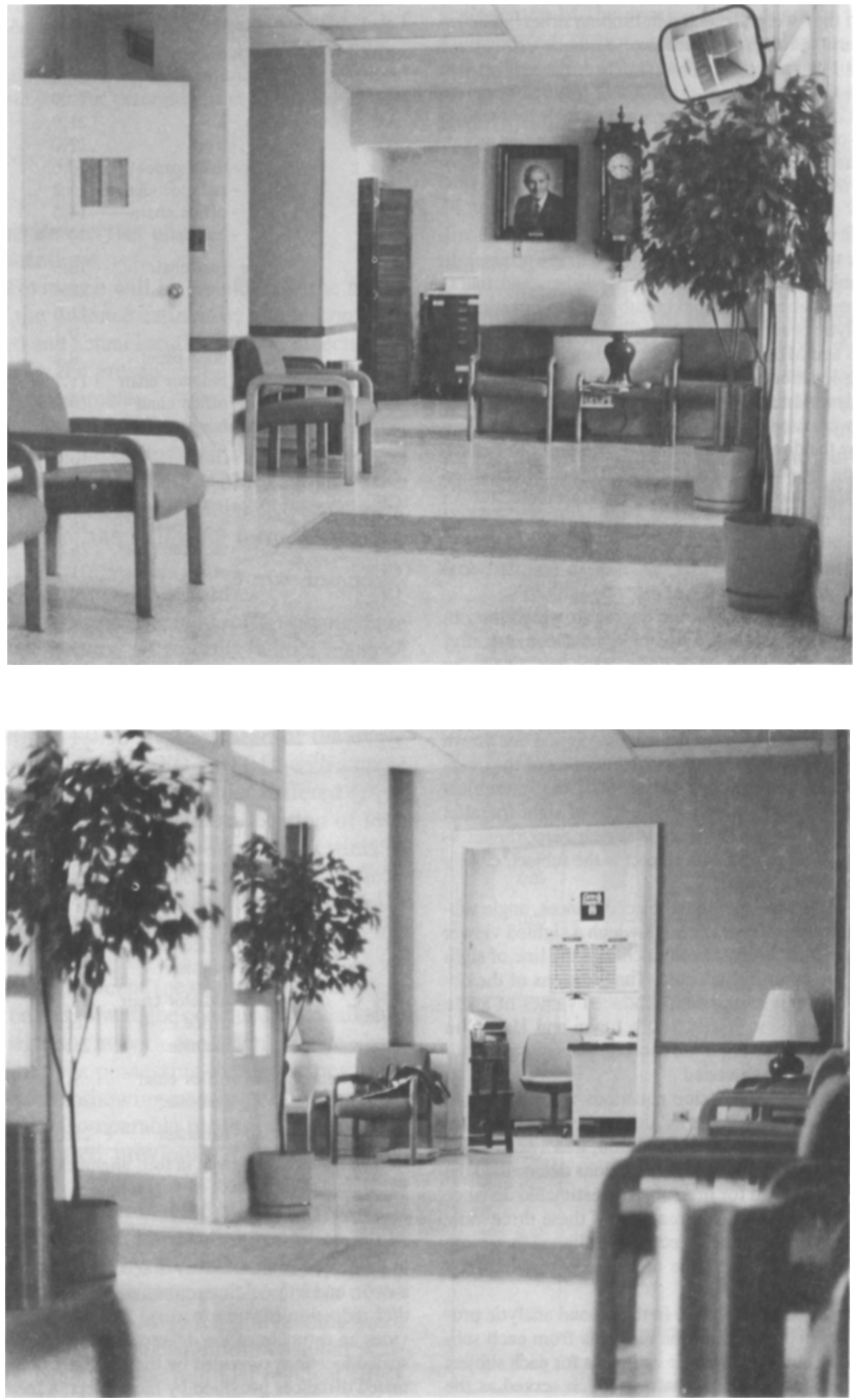

Figure 2. Two views of the experimental room. The top view is taken from the desk at the west end of the room, looking toward Objects $C$ and $D$. Object $A$, where the subject sat, is at the extreme left of the picture, just to the len of the door to the corridor. The bottom view is taken from the east end of the room, next to Object C, looking toward the desk at the west end of the room. 
continued in order on the data sheet. The order of the 45 pairs on the data sheets was arranged thus: $A B, A C, \ldots A J, B C, B D, \ldots$ $\mathrm{BJ}, \mathrm{CD}, \mathrm{CE}, \ldots \mathrm{CJ}, \ldots \mathrm{IJ}$. (Pilot testing indicated that the blind subjects had difficulty keeping track of the correct pair of objects to be judged when they were presented in random order.) The instructions, familiarity testing, and absolute distance estimations usually took about $1 \mathrm{~h}$ altogether. When finished, the subject was asked to return on the next day for the in-room testing. These 14 subjects produced 28 sets of data, 1 from each of their two testing sessions. For each of the 6 subjects in the sighted-unfamiliar group, all of the testing was done in a single session in the lobby experimental room, so that they produced six data sets.

\section{Analytic Procedures}

These data were used both to confirm that distance estimates do provide a reasonable reflection of spatial knowledge, and to extract a spatial representation that could be subjected to quantitative analysis. Two analytic procedures were applied to the $\mathbf{3 4}$ data sets: regression analysis and MDS. In each case, the data from each subject in each testing location were scored and analyzed separately.

Regression analyses. In the first analytic procedure, the absolute distance estimates were used directly. Table 1 lists the $\mathbf{4 5}$ interobject distances formed by the direct line distances between all pairs of the 10 objects (counting the subject as an object). These are classified on three variables that, according to the research literature, reflect possible distortions in the perceived scene and predict estimated absolute distance: true distance, alignment, and visual angle.

The first classification lists the true distance, in feet, between the objects in each pair.

The second class, alignment, lists the degree to which the objects in the pair are aligned with the line of sight. Geometrically, this corresponds to the orientation, in degrees, of the line connecting each pair of objects with respect to the subject's viewing position. The alignment of an interobject distance is defined by the angle formed between a line drawn between the two objects and one drawn along the line of sight from the subject to the midpoint of the interobject distance. When the angle approaches $90^{\circ}$, the interobject distance approaches perpendicularity to the line of sight (parallel to the horizon). In contrast, when the angle is near zero, the interobject distance is radially oriented with respect to the subject, closely resembling the line of sight itself.

Finally, the third classification of interobject distances, angle subtended, lists the angle, in degrees, through which a sighted viewer would have to turn his/her eyes or head to change the line of sight from one object to the other in each pair. The locations of the objects and the subject in this experiment produced values of angle subtended ranging from $0^{\circ}$ to nearly $160^{\circ}$. Levin and Haber (in press) provide further details about the scoring of alignment with the line of sight and angle subtended.

Both nonlinear and linear regression equations were computed for each subject for each of the subjects' data sets, one set for the 45 estimates made in the remote room condition, and a second for those made in the in-room condition. The equations determined the variance that can be accounted for in a subject's estimated distance between the objects in each pair as a function of these three variables: the true distance between the objects; how aligned they were with the line of sight; and how large an angle subtended they required.

Multidimensional scaling analyses. In the second analytic procedure, MDS was applied to each of the data sets from each subject. Each set of the $\mathbf{4 5}$ absolute distance estimates for each subject was entered directly into a diagonal matrix, which served as the input. The MDS program finds the best locations of the 10 objects relative to each other such that differences among the 45 estimated interobject distances and the $\mathbf{4 5}$ scaled distances are minimal. ${ }^{2}$

Two measures were derived from the output of each MDS solution. The first MDS measure concerns the internal consistency of the subject's judgments, shown by the amount of residual stress
Table 1

Classification of the 45 Interobject Distances Among the 10 Objects Shown in Figure 1

\begin{tabular}{|c|c|c|c|c|}
\hline Label & Names & $\begin{array}{c}\text { True } \\
\text { Distance }\end{array}$ & Alignment & $\begin{array}{c}\text { Angle } \\
\text { Subtended }\end{array}$ \\
\hline $\mathrm{AB}$ & self-hinge & 18.4 & 0.0 & 0.0 \\
\hline $\mathrm{AC}$ & - east chair & 20.0 & 0.0 & 0.0 \\
\hline $\mathrm{AD}$ & - cart & 29.9 & 0.0 & 0.0 \\
\hline $\mathbf{A E}$ & $-T V$ & 21.9 & 0.0 & 0.0 \\
\hline $\mathrm{AF}$ & -tree & 12.0 & 0.0 & 0.0 \\
\hline $\mathrm{AG}$ & -door panel & 10.6 & 0.0 & 0.0 \\
\hline $\mathrm{AH}$ & - radiator chair & 16.2 & 0.0 & 0.0 \\
\hline AI & - office chair & 14.5 & 0.0 & 0.0 \\
\hline AJ & -bannister & 14.7 & 0.0 & 0.0 \\
\hline $\mathrm{BC}$ & hinge-east chair & 18.5 & 84.2 & 57.2 \\
\hline $\mathrm{BD}$ & - cart & 28.4 & 61.3 & 67.2 \\
\hline $\mathrm{BE}$ & $-\mathrm{TV}$ & 11.6 & 71.8 & 32.0 \\
\hline $\mathrm{BF}$ & -tree & 9.1 & 43.9 & 25.1 \\
\hline BG & -door panel & 8.1 & 13.3 & 8.0 \\
\hline BH & - radiator chair & 11.1 & 78.0 & 36.5 \\
\hline BI & - office chair & 19.5 & 75.9 & 71.5 \\
\hline BJ & - bannister & 29.1 & 75.0 & 122.5 \\
\hline CD & east chair-cart & 10.7 & 22.3 & 9.7 \\
\hline $\mathrm{CE}$ & $-\mathrm{TV}$ & 9.5 & 78.5 & 25.7 \\
\hline CF & - tree & 11.9 & 45.2 & 32.8 \\
\hline $\mathrm{CG}$ & -door panel & 15.4 & 47.8 & 49.2 \\
\hline $\mathrm{CH}$ & - radiator chair & 26.9 & 77.3 & 95.0 \\
\hline CI & - office chair & 31.2 & 67.2 & 128.0 \\
\hline $\mathrm{CJ}$ & -bannister & 19.2 & 70.9 & 65.2 \\
\hline DE & cart-TV & 17.8 & 61.8 & 36.0 \\
\hline $\mathrm{DF}$ & - tree & 22.6 & 33.0 & 42.8 \\
\hline DG & -door panel & 26.2 & 34.9 & 59.8 \\
\hline DH & - radiator chair & 37.2 & 56.3 & 103.4 \\
\hline DI & - office chair & 41.8 & 40.7 & 137.3 \\
\hline DJ & -bannister & 23.7 & 45.4 & 51.3 \\
\hline EF & TV-tree & 10.1 & 16.7 & 6.5 \\
\hline EG & -door panel & 12.7 & 24.6 & 26.4 \\
\hline EH & - radiator chair & 20.0 & 70.8 & 61.0 \\
\hline EI & - office chair & 28.4 & 66.6 & 100.3 \\
\hline EJ & - bannister & 26.4 & 67.8 & 90.0 \\
\hline FG & tree-door panel & 3.6 & 66.4 & 16.8 \\
\hline FH & - radiator chair & 14.9 & 70.7 & 61.4 \\
\hline FI & - office chair & 22.1 & 78.4 & 112.4 \\
\hline FK & - bannister & 20.2 & 78.2 & 97.8 \\
\hline GH & door panel-radiator chair & 11.5 & 57.9 & 44.9 \\
\hline GI & -office chair & 16.1 & 71.9 & 78.0 \\
\hline GJ & - bannister & 21.4 & 69.6 & 114.6 \\
\hline HI & radiator chair-office chair & 9.3 & 78.6 & 34.3 \\
\hline HJ & -bannister & 30.6 & 73.0 & 161.3 \\
\hline $\mathbf{U}$ & office chair-bannister & 28.6 & 87.6 & 156.8 \\
\hline
\end{tabular}

Note-Distances are given in feet; alignment with the line of sight and visual angles subtended, in degrees.

in the scaled solution. This is accomplished by attempting a one-, a two-, and a three-dimensional solution, each iterated until no further reduction in stress occurs. The resulting residual stress provides an estimate of the degree to which the distances among the scaled locations provided by the MDS are consistent with the estimated distances provided by the subject, a measure of the internal organization of the subject's estimates.

The second MDS measure concerns the subject's accuracy in locating the actual objects. This is found by examining the degree to which the scaled locations determined by MDS among the 10 objects resembles the actual arrangements among those objects in the scene. To determine this, the derived coordinates of each ob- 
ject are compared with their true coordinates. An initial configuration made up of the true coordinates of the objects is provided to the MDS program. The amount of stress between the two sets of coordinates is determined before any iterations are made. ${ }^{3}$

After the regression equations and the MDS solutions were computed for each condition of each individual subject's data, the appropriate derived values, coefficients, or constants were averaged within or between subjects for descriptive and inferential statistical analyses.

\section{RESULTS}

\section{Evidence for the Properties of \\ Spatial Representations}

Three kinds of evidence will be considered: the metrical properties of the distance estimates; global comparisons among scaled and actual locations of the objects; and distortions shown by the presence of other variables that predict the distance estimates.

Metrical properties. To replicate evidence that sighted subjects have a ratio scale of distances among objects in near space, and to examine whether blind subjects also have a ratio scale of distances, we computed 34 nonlinear regression equations of the following form:

$$
\text { estimated distance }=\text { intercept }+(\mathrm{T}) \text { true distance }{ }^{t} \text {. }
$$

The proportion of variance accounted for $\left(R^{2}\right)$ among these 34 equations ranged from 0.85 to 0.99 , with a mean of $0.95(S D=0.05)$. The mean of the intercept was $+0.02 \mathrm{ft}(S D=0.33)$, with a range over the 34 equations from -1.10 to $+0.95 \mathrm{ft}$. The mean of the exponent $t$ on true distance was $0.97(S D=0.04)$, with a range of 0.90 to 1.08 . None of these variables differed ( $p>$ $.20)$ between the subject groups or the location of testing. There was substantial variation among the estimates of the regression coefficient on true distance $(T)$, reflecting differing degrees of over- and underestimation. These were correlated with the independent variables and are reported below.

The results for the intercept (near zero) and exponent (near unity) are consistent with the conclusion that all subjects, blind and sighted, in both conditions, made absolute distance estimations possessing the properties of an underlying ratio scale, showing nearly perfect linearity and a true zero point. Comparable results on sighted subjects have been reported previously by Toye (1986), Przeorek (1986), and Levin and Haber (in press). The results reported here indicate that the blind can make absolute distance estimates that have ratio properties, quite comparable to those made by the sighted.

Global comparisons. The most general evidence of the quality of a subject's spatial knowledge available from this experiment comes from the multidimensional scalings. One analysis examines the minimal residual stress in using the subject's estimates to locate the objects in a scaled map of the experimental space. Another analysis compares the locations of the 10 objects in the scaled map space with their true locations.

Internal consistency of the scaled maps. If a subject's set of responses is consistent with some scene (regard- less of its resemblance to the real scene in this experiment), the MDS program would have no difficulty in using all of the estimates of that subject to construct a scaled map of the space. Three tests of this consistency are reported: the amount of residual stress remaining after a large number of iterations; the number of dimensions required to minimize stress; and the number of particularly difficult points to fit.

The residual stress results are shown in the lower portion of Figure 3. The mean residual stress (averaged over 34 solutions) was 0.050 when the scaling was done in two dimensions, with a range from 0.122 to 0.006 . Even the highest (poorest) values of stress were well within the region that Kruskal (Kruskal \& Wish, 1978) reserved for very high consistency of fits of the judgments to the scaling. Thus, all subjects in all conditions were able to produce judgments that could easily be scaled.

The number of dimensions needed to represent the scaled map of the space should be two, since the distances among the objects are physically measured along a single plane of two dimensions. The mean residual stress, averaged over all 34 scaled maps, was 0.281 for one dimension, 0.050 for two dimensions, and 0.034 for three dimensions. Thus, the internal consistency was nearly at

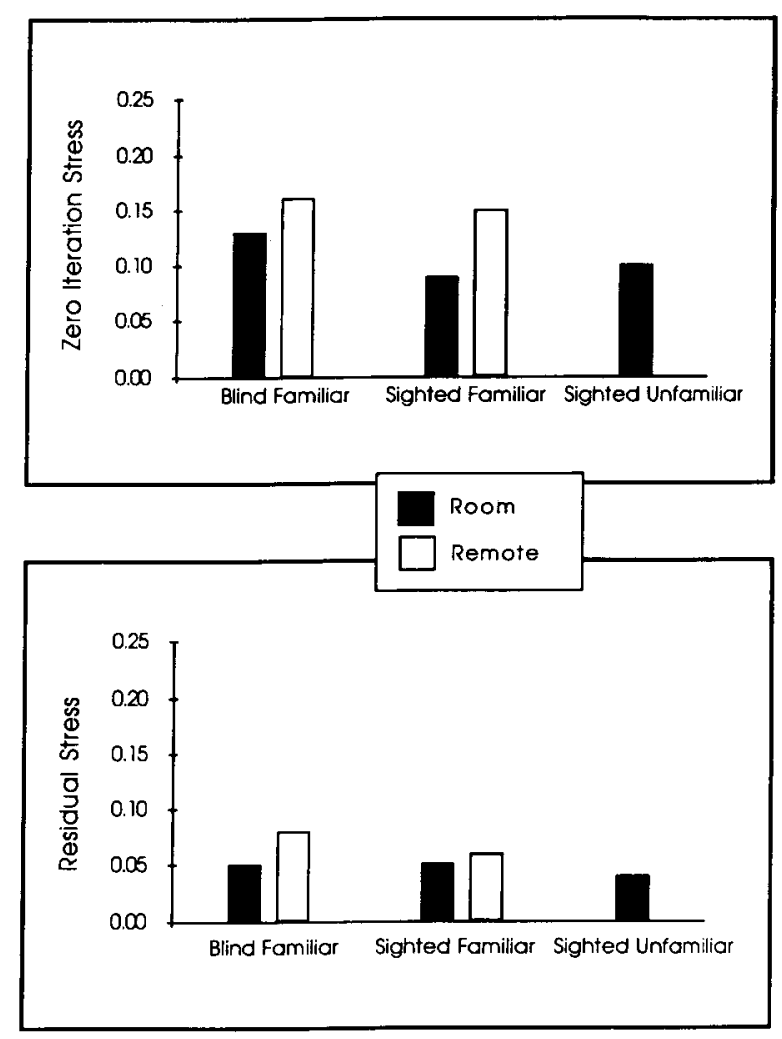

Figure 3. The top panel shows the mean uniterated stress (a measure of the resemblance of the subjects' judgments to the true values in the actual scene) as a function of familiarity and visual status, for both the in-room and the remote testing. The bottom panel shows the residual stress (a measure of the consistency of the subjects' judgments) as a function of the same independent variables. 
a minimum at two dimensions, and adding a third (or additional) dimension did not significantly reduce the stress of the final solution any further. By Kruskal's elbow test (Kruskal \& Wish, 1978), the scaled solutions based on two dimensions represented the data most consistently.

Finally, in none of the $\mathbf{3 4}$ scaled mappings were there any points that the MDS program had difficulty in scaling.

Accuracy of spatial knowledge of the actual scene. The goodness of fit of the scaled map of the space to the real space was assessed by providing an initial configuration for the MDS based on the coordinates of each of the objects in the real scene and examining the resulting stress between the initial configuration and the scaled configuration before any iterations were made. If each of the objects in the map of the scaled scene had the same respective coordinates as those in the true scene, the initial stress without any iteration would be zero.

The before-iteration stress results are shown in the upper portion of Figure 3. The mean stress before iteration, based on the seeded coordinates of the true locations, was 0.125 (averaged over all 34 scaled maps), with a range among the 20 subjects of 0.039 to 0.228 . Again, even the poorest stress values for the goodness of fit were below the criterion Kruskal proposed for assessing very good fits.

Another indication of the match of a spatial representation to the actual scene is shown by the amount of variance in the distance estimate accounted for by the true distances among the objects. If the proportion of variance accounted for approaches unity, using just the single predictor of true distance, this suggests that the distance estimates reflect an underlying, accurate representation of the space.

Given the evidence of linearity already reported, we used the following linear regression equation for each of the 34 sets of data:

$$
\text { estimated distance }=\text { intercept }+(\mathrm{T}) \text { true distance. }
$$

The variable of true distance by itself accounted for nearly all of the variance in predicting estimated distance in each of the three groups, and in each of the two testing locations. The $R^{2}$ values ranged from 0.84 to 0.99 , with a mean over the 34 equations of $0.94(S D=0.052)$. There were no differences in $R^{2}$ among or within groups. These proportions are very high, suggesting that not much variance remains for any other variable, distorting or otherwise.

The intercept constant did not account for any significant variance, in that its regression weight never differed significantly from zero for any subject in any group or condition. Therefore, as a group, subjects in this experiment provided evidence of spatial representations that were internally consistent, that globally resembled the true space being judged, and that specifically reflected the actual distances among the objects in the scene. Further evidence of the ratio scaling of distance is shown by the virtual identity of the findings based first on nonlinear and then on linear regression equations.
Distortions. So far, the results have indicated that the subjects' responses were consistent with very accurate spatial representations. The evidence in the literature of distortion by sighted subjects has suggested an elongation of represented space in comparison with the actual scene, as indicated by significant effects of alignment and visual angle subtended variables in predicting estimated distance.

To test for these effects in the present experiment, regression equations were used to examine the impact of these addtional predictor variables. No distortions would be indicated if these additions were statistically insignificant. The complete equation tested was as follows:

$$
\begin{aligned}
\text { estimated distance }= & (\mathrm{T}) \text { true distance }+(\mathrm{A}) \text { alignment } \\
& +(\mathrm{VA}) \text { visual angle subtended. }
\end{aligned}
$$

The significance of each predictor was separately determined by a stepwise procedure. We computed the proportion of variance accounted for $\left(R^{2}\right)$ by the regression equation, using just true distance as the single predictor (reported above), and then we added each of the other predictors, one at a time, and examined the significance of the improvement in $R^{2}$.

The addition of the variables of alignment and visual angle subtended had no significant effect in any equation from data collected in the remote testing room, or for any equation from blind subjects in either testing setting (all ps $>.20$ ). However, adding alignment increased the proportion of variance accounted for in both groups of sighted subjects in the in-room testing [mean $\mathrm{A}=+.03(S D=$ $.008), p<.01]$. Adding visual angle subtended also increased the predictability for the sighted subjects in the in-room testing, but only for the sighted familiar subjects [mean VA $=-.02(S D=.008), p<.01$ ]. Surprisingly, the visual angle subtended predictor was not different from zero in the sighted unfamiliar group $(p>.20)$.

Finally, if we consider the 34 equations that included all the variables, the proportion of variance accounted for $\left(R^{2}\right)$ in predicting estimated distance by these variables ranged from 0.90 to 0.99 , with a mean of $0.97(S D=$ .031 ). There were no significant differences between or within groups.

Though not a measure of distortion, changes were found across groups and conditions in the amount of over- and underestimation of distances, as given by the slope of the regression equation, indicated by regression weight on true distance $(T)$ - -see Figure 4. A true distance weight of 0.90 indicates that each estimated distance is consistently $90 \%$ of true distance-a $10 \%$ underestimation.

When tested in the room, the sighted familiar and sighted unfamiliar groups showed insignificant underestimations of $5.0 \%$ and $5.1 \%$, respectively. The blind group significantly underestimated distance by $12.2 \%$ ( $p<$ $.001)$, which was also significantly greater than either of the sighted groups $(p<.01)$. However, when tested in the remote room, both the sighted and the blind groups underestimated distance by $29.5 \%$ and $30.7 \%$, respectively, which were significantly greater amounts of under- 


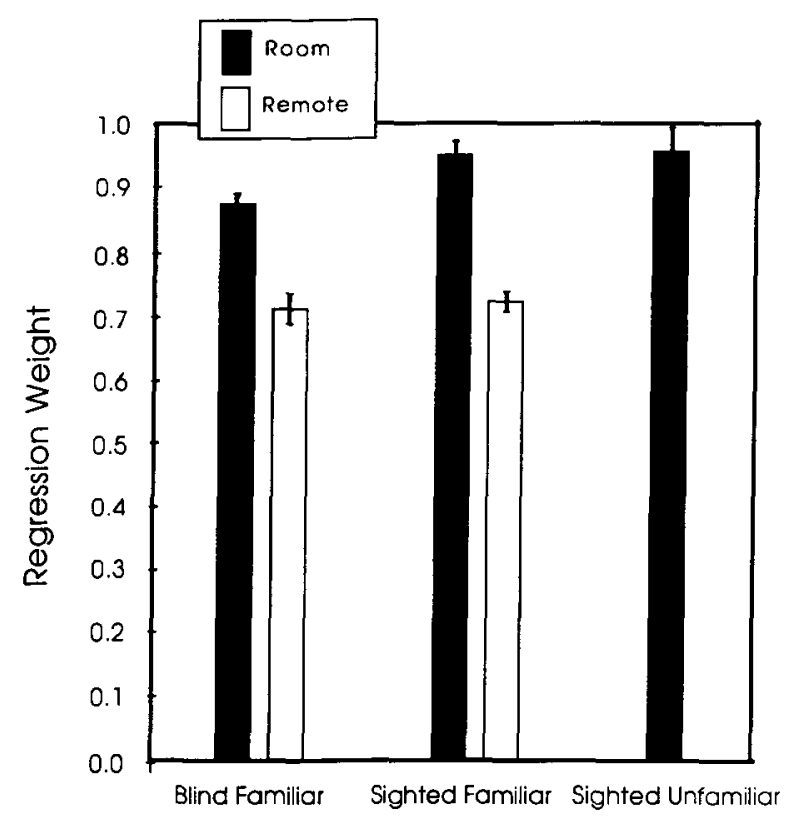

Figure 4. The mean regression weight $(T)$ on true distance (an index of the slope of the regression equation, and a measure of the amount of over- or underestimation of distance), as a function of familiarity and visual status, for both the in-room and the remote room testing. Standard deviations are indicated at the top of each bar.

estimation in comparison with the respective in-room testing scores $(p<.001)$, but not significantly different from each other. Therefore, these experimental variables caused subjects to change the scaling constants that they used to estimate distance. This produced, for all subjects, a smaller represented scene when they were tested in the remote setting as opposed to the in-room testing.

To summarize the distortion results-all subjects provided relatively undistorted spatial representations under all conditions examined. However, the sighted subjects tested while looking at the scene produced slight distortions, suggesting an elongation along the dimension perpendicular to the line of sight in their representations of the scene. All subjects had considerably smaller spatial representations when tested in a remote room.

\section{Effect of Scene Familiarity}

This effect was assessed by comparisons between the subject groups who were familiar with the lobby (both sighted and blind) and those who were not (sighted only). In no analysis did familiarity show any significant impact on the spatial representations. In the MDS analyses, familiarity with the experimental scene did not significantly affect either the amount of internal consistency $(t=$ $1.306, p>.20$-see Figure 3, bottom), or the accuracy of the scaling to the actual scene $(t=0.964, p>.40-$ see Figure 3, top). In the regression analyses, neither the percentage of variance accounted for nor any of the regression weights differed as a function of familiaritysee Figure 4. These negative findings also held when only the two sighted groups were compared.

\section{Effect of Testing Location}

If we consider the MDS analyses of the 14 subjects in the two familiar groups ( 7 blind and 7 sighted) who were tested twice, judgments made in the experimental room showed significantly better fits to the real scene (mean uniterated stress was 0.114 vs. $0.142 ; t=2.989, p<$ .004 -see Figure 3 , top), and significantly better internal consistency (mean residual stress was 0.044 vs. 0.058 ; $t=2.124, p<.038$-see Figure 3, bottom), in each case when compared with judgments made in the remote room. Neither of these two differences interacted significantly with visual status, so that the slight benefit shown by the MDS analyses from testing in the experimental space was found for both sighted and blind subjects equivalently.

The magnitude of the difference between in-room and remote testing was about the same within the blindfamiliar group alone as within the sighted-familiar group alone, but the differences for each group alone no longer met appropriate significance levels, presumably because of the smaller number of subjects. Hence, we can only accept very tentatively this effect for the two subject groups separately.

Two relevant results from the regression equations pertaining to the testing location have already been reported. First, sighted subjects show some elongation in their spatial representations when tested in the room, an effect that disappears in the remote testing. Second, all subjects underestimate distance more in the remote setting, as is shown in Figure 4.

\section{Comparability of Sighted and Blind Subjects}

If we consider the MDS analyses first, the subjects in the two sighted groups $(n=13)$ had significantly better agreement between their scaled maps and the true locations of the objects than did the blind-familiar subjects $(n=7)$ : mean uniterated stress was 0.116 versus 0.141 , respectively ( $t=2.539, p<.014-$ see Figure 3, top). The subjects in the two sighted groups also had scaled mappings that were more internally consistent than those of the blind-familiar subjects: mean residual stress was 0.041 versus 0.065 , respectively $(t=3.805, p<.001-$ see Figure 3, bottom). Although these differences were statistically reliable, they were not especially large.

Sightedness and location of testing did not interact significantly. Thus, within this study, the slightly better representations of the sighted subjects in comparison with those of the blind subjects was equivalent when the testing was done in the room and when it was done remotely.

The regression equation analyses showed very little difference between sighted and blind subjects: they both produced equivalent ratio scaling of distances; and their equations predicting estimated distances accounted for equivalently high amounts of variance. Only in terms of the amount of underestimation did the blind differ from the sighted, but again, the effect is not particularly large, as is shown in Figure 4.

The 7 blind subjects were examined separately to determine whether age of onset of blindness or totality of blindness was correlated with any of the preceding dif- 
ferences. Statistically, dividing the subjects into 2 congenital as opposed to 5 late blind (or 3 early blind as opposed to 4 adult blind) revealed no trends of any kind: the early blind provided characteristics of spatial knowledge comparable to those for the later blind. The same result was found by dividing subjects into total blind opposed to light perception only. There was no hint that adding more subjects would have improved significance levels-there were no trends at all.

\section{DISCUSSION}

\section{Properties of Spatial Representations}

The strongest evidence for an accurate internal spatial representation, one that mirrors the true scene, comes from the finding that the subjects' distance estimates reveal an underlying ratio scale of distance. This shows that these subjects, blind and sighted, are able to judge all of the relationships within the scene, using the same scale of space. Our results could not have been stronger had we given our subjects a yardstick and asked them physically to measure these distances and then report them. This was true even when subjects made the judgments in the remote testing setting. All that changed with remote testing was that the hash markings on this hypothetical ruler were farther apart.

These results replicate the previous work of Toye (1986), Wagner (1985), Przeorek (1986), and Levin and Haber (in press)- -especially the demonstrations of ratio scaling of space. These results also extend the findings to an indoor scene, to estimates made without concurrent visual experience of the scene, and to blind subjects.

Evidence was found, however, for small, systematic distortions by the sighted subjects when they made their responses during direct viewing. These distortions also replicate previous findings under comparable conditions. Levin and Haber (in press) speculate that this distortion might be related to the magnitude of actual eye and head turns made during the viewing of the objects. This speculation is entirely consistent with the results here: the distortions were absent in subjects tested from memory, where eye and head turning would be uncorrelated with information processing; and they were absent in the blind under either testing condition. Thus, these distortions, even though small (and not even consistently found in all sighted subjects), do suggest some small nonlinearities in the visual perception and processing of space (see Levin \& Haber, in press, for a discussion).

Following Siegal and White (1975), one might predict that within the distance estimation data from blind subjects, interobject distances coinciding with direct, unobstructed routes between objects would be estimated more accurately than those between objects requiring circuitous travel. We attempted to test this prediction in the present experiment by dividing the 45 interobject distances into those permitting straight-line travel and those not. There were no differences, which may not be surprising, given that the very high $R^{2}$ s left little room to demonstrate the impact of other variables. The lack of difference may also reflect the high level of travel skills of these blind subjects.

\section{Familiarity}

Prior familiarity among sighted subjects does not affect the spatial representations made by the sighted when looking at the scene. Distance estimates themselves do not change as a function of familiarity, nor does the degree to which those estimates can be scaled to match the true scene. In general, these data suggest that the familiarity of sighted subjects with the scene when they are looking at the scene while making their judgments is unimportant in the perception of spatial layout.

Although this scene was unfamiliar to one group of subjects in this experiment, it was not atypical of their prior experience. These data do not tell us whether spatial representations of a scene that is unfamiliar and unusual would also be accurate. Following Tversky's (1981) findings that spatial representations of maps retained in memory are inaccurate in that they are regularized toward typical expectations, we might expect comparable systematic inaccuracies during direct viewing of a scene that is unfamiliar because it is atypical. This remains to be tested.

We included the familiarity variable in the sighted because it is typically confounded in the literature when one attempts to compare across experiments in which sighted and blind subjects have participated. As a result of our experiment, we can now conclude that whatever differences may be found between the spatial representations of the blind and the sighted, these differences do not result from differential familiarity with this kind of scene. Such differences between the blind and the sighted would obtain even in the comparison of judgments made about a highly familiar scene by blind subjects with judgments made about a comparable scene never before experienced by sighted subjects.

The present experimental design did not permit examination of the effect of scene familiarity among blind subjects, nor among sighted subjects tested from memory. It seems obvious that all subjects would display poorer spatial knowledge about a scene they knew less about whenever they were tested without concurrent sensory information about the scene. However, the nature of the impact of familiarity remains to be demonstrated in cases when there is no sensory input from the scene available to inform or guide the spatial responses being made, for either blind or sighted subjects. The critical finding here is that with concurrent sensory processing, sighted subjects do not benefit from prior familiarity with at least this kind of scene.

\section{Concurrent Viewing Versus Memory}

Before we discuss the implications of the testing location differences in our results, we need to consider whether the order of testing might have confounded these conclusions. For experimental convenience, all 14 subjects in the two familiar groups were tested in the remote room first, and then tested a second time in the experimen- 
tal room. Although it is possible that the differences between remote and in-room findings are due to order effects that were manifested through the benefit of practice, the cost of boredom, or the opportunity to check the true distances between testings, none of these explanations seems likely.

First, when Toye (1986), Przeorek (1986), and Levin and Haber (in press, Experiment 1) assessed some or all of their sighted subjects twice under comparable conditions, they found no evidence across replications of either a practice effect or a boredom effect. In neither regression nor scaling analyses did subjects systematically change between the two testings. Second, in the present experiment, the results from the first (and only) in-room testing session of the sighted-unfamiliar subjects matched those of the second in-room testing session of the sighted-familiar subjects in all respects, so the latter were not showing any benefit of practice, or cost of boredom (these comparisons provided the negative evidence concerning any possible familiarity effect). Third, there was no hint in the postexperimental debriefings that any of the subjects suspected that their responses in the two testing conditions might have differed in any way, or that they had checked out the experimental space between testings, or paced off any of the distances. Consequently, we are comfortable in interpreting the differences between these two testing conditions as being attributable to the nature of the conditions themselves, and not to any confounding of order, practice, boredom, or opportunity to verify the space.

Two differences as a function of testing location were shown for all subjects in the present experiment: a small increase in the MDS's stress in the remote location, and a substantial increase in underestimation of distance. The first finding (increase in MDS stress) suggests a slight loss in accuracy or precision, probably attributable to error variance, though without any loss in precision of scaling true distance to estimated distance. The second increase (increase in underestimation) suggests that the spatial representation of the size of remembered space is smaller than the representation of the size of concurrently experienced space. Wiest and Bell (1985) have reported a comparable finding: underestimation of distances (and lower power functions) are found for remembered distances in comparison with directly viewed distances. Although nonlinearity (the power of the function) did not increase under remote testing in this experiment, underestimations (the slope of the function) certainly did.

Note that the underestimation effect is indexed by the magnitude of the regression coefficient on true distance, not by an error term. The subjects are just as precise in their judgments of distance and are still using a ratio scale of space-they have only changed the scaling constant.

Had these differences between the two testing locations been restricted only to the sighted subjects, it would suggest the interpretation that the experimental manipulation simply reflected the difference between reporting while looking and reporting while remembering (on the assumption that blind subjects, even while in a scene, do not have access to the global perceptual information available to the sighted through vision).

However, the blind-familiar group also showed comparable differences between the two testing locations, so the equation of the sighted with concurrent perceiving and the blind with memory is too simplistic.

It has been reported in other studies that blind subjects provide less accurate data when they are tested remotely than when they are tested in the space (e.g., Rieser et al., 1986). At least two alternative explanations for this difference can be offered. The first is that the blind subjects, like the sighted, receive and can process global sensory information when they are tested in the room that is unavailable to them when tested in the remote testing location. It is known that blind people, even when stationary, receive spatial information from the patterns of auditory stimulation that define spatial volume, boundary walls, and even object locations, and from tactile stimulation arising from air currents. These can specify both individual objects and global properties of the relationships among many objects distributed within the volume of the scene (see Hill \& Ponder, 1976, for examples). To this extent, both the blind and the sighted may have been picking up equivalent information from the scene when they were physically seated in the test space. This would account for their equivalent performance in the room, and their equivalent loss in accuracy when they were tested remotely. We collected no postexperimental evidence regarding the possibility that subjects may have been picking up and processing concurrent sensory information during the in-room testing. Even if this were the case, it would be difficult to understand why the absence of such sensory information could cause such systematic underestimation of distances.

An alternative, more cognitive explanation, is often offered for the in-room versus remote differences: being in the space makes it easier to access its stored spatial representation. Sighted people (and many blind persons as well) might say that it is easier to "visualize" or imagine the scene when one is actually there, even in the absence of any concurrent sensory stimulation. Again, difficulty in remote visualization would apply to both the sighted and the blind. This cognitive interpetation goes well beyond a simple memory versus sensory information processing distinction. To pursue this explanation, it is necessary to determine why increasing the difficulty of access to one's spatial representation of a scene makes that representation smaller.

Unfortunately, the present experiment provided no manipulations to distinguish between these alternatives.

Finally, with respect to the effect of location of testing, one difference was reported in which the sighted subjects showed a small distortion in their spatial representations when tested in the room that was not found in the remote testing (and was not found in the blind subjects in either testing location). Because this finding is restricted to the sighted subjects, and then only to the in-room testing, it is most easily interpreted as the result of concurrent visual processing of sensory stimulation. 


\section{Comparability of the Sighted to the Blind}

Our results show that all of the subjects in this experiment had spatial representations of the test scene that accurately reflected its spatial properties. This was equally true for the blind, including the 2 congenitally blind who had never experienced any visual interaction with their environment. In this critical respect, the spatial representations of the sighted and the blind are qualitatively comparable: the small statistical differences in MDS stress or underestimation do not even introduce much quantitative dissimilarity.

The blind subjects in this experiment showed consistently more accurate spatial representations than have generally been reported in the literature. However, these subjects are not typical of those usually found in research on blind subjects: their independent travel experience ranged from 10 to 30 years.

These results suggest to us that quality and amount of travel experience and other interactions with the environment are a more important predictor of the accuracy of spatial representations of that environment than present visual status or amount of previous visual experience (Haber \& Haber, 1993, extend this argument). The present study examined the abilities of adult blind observers who regularly use well-developed travel skills. Lockman et al. (1981) and Rieser et al. (1992) provide direct empirical support for the importance of the role of travel skill where such skill was more varied in the subject populations studied. Loomis et al. (in press) also report data from several navigation tasks, in which no differences were found between blind and sighted subjects. However, their subject groups were not comparable in terms of several other important variables, making the lack of a difference difficult to interpret (see Haber \& Haber, 1993, for further discussion of those results).

The results reported here also suggest that the spatial representations of these blind subjects resemble those of the sighted both qualitatively and quantitatively. Therefore, these results provide evidence that neither recent nor any visual experience is necessary for the development and functioning of high levels of spatial organization, the concept of spatial layout, the notion of a person's viewpoint, or a metrical knowledge of the environment. Although it has been concluded in important studies that visual blindness does not necessarily prevent acquisition and performance of principles of spatial organization (e.g., Heller \& Kennedy, 1990; Landau, 1988), the present results provide this evidence in a broader context.

This conclusion is also in contradiction to recent work by Lederman (e.g., Lederman, Klatzky, \& Barber, 1985), who have suggested that blind persons, because of their heavy use of tactile exploration and processing, would be especially proficient at three-dimensional representations, and poor at tasks involving properties of two-dimensional arrangements. The present experiment demanded only two-dimensional processing and showed no deficiency in the blind subjects, either in contrast to the sighted, or with respect to the true layout of the scene being judged. One finding of a difference between sighted and blind groups was uncovered here: distortions made by the sighted during direct viewing that were not present in the data from the blind. As discussed earlier, this difference can be attributed to the concurrent visual process itself, rather than to some fundamental difference between sighted and blind subjects.

\section{Methodology}

As a final item of discussion, we selected the absolute distance response measure for its power and its ease to elicit and score, and because pilot testing indicated that blind subjects could use it readily. The blind subjects in the actual experiment were as good at using the measure as were our sighted subjects. While this is the first report of data from blind subjects using this method, this success suggests that the method is appropriate for use in other experiments with blind subjects.

\section{REFERENCES}

Brambring, M. (1985). Mobility and orientation processes of the blind. In D. H. Warren \& E. R. Strelow (Eds.), Electronic spatial sensing for the blind (pp. 493-508). The Hague: Martinus Nijhoff.

Foulke, E. (1985). The cognitive foundations of mobility. In D. H. Warren \& E. R. Strelow (Eds.), Electronic spatial sensing for the blind (pp. 463-486). The Hague: Martinus Nijhoff.

HABER, R. N. (1985a). Toward a theory of the perceived spatial layout of scenes. Computer Vision, Graphics \& Image Processing, 6, 282-321.

HABER, R. N. (1985b). Understanding perceived spatial layout of scenes: A prerequisite for protheses for blind travelers. In D. H. Warren and E. R. Strelow (Eds.), Electronic spatial sensing for the blind (pp. 431461). The Hague: Martinus Nijhoff.

HABER, R. N. (1990). Dynamic stimuli and active perceiving in human visual perception. In Sensor fusion II: Human and machine strategies (pp. 3-29). Bellingham, WA: The Society of Photo-Optic Instrumentation Engineers.

HABER, R. N., \& HABER, L. (1991). Why mobile robots need a spatial memory. In Sensor fusion III: Human \& machine strategies (pp. 815823). Bellingham, WA: The Society of Photo-Optic Instrumentation Engineers.

HABER, R. N., \& HABER, L. (1993). Measuring the invisible: Spatial representations and their assessment. Manuscript submitted for publication.

Heller, M. A., \& Kennedy, J. M. (1990). Perspective taking, pictures, and the blind. Perception \& Psychophysics, 48, 459-466.

Hul, E. W., \& Ponder, P. (1976). Orientation and mobility techniques: A guide for the practitioner. New York: American Foundation for the Blind.

HollyField, R. L., \& Foulke, E. (1983). The spatial cognition of blind pedestrians. Joumal of Visual Impairment \& Blindness, 9, 204-210.

Kosslyn, S. M., Pick, H. L., JR., \& Farello, G. R. (1974). Cognitive maps in children and men. Child Development, 45, 707-716.

Krumhansl, C. L. (1979). The psychological representation of musical pitch in a tonal context. Cognitive Psychology, 11, 346-374

Kruskal, J. B., \& Wish, M. (1978). Multidimensional scaling. London: Sage University Papers.

LANDAU, B. (1988). The construction and use of spatial knowledge in blind and sighted children. In J. Stiles-Davis, M. Kritchevsky, \& U. Bellugi (Eds.), Spatial cognition: Brain bases and development (pp. 343-371). Hillsdale, NJ: Erlbaum.

Lederman, S. J., Klatzky, R. L., \& Barger, P. (1985), Spatial- and movement-based heuristics for encoding pattern information through touch. Journal of Experimental Psychology: General, 114, 33-49.

LEVIN, C. A. (1992). A case against the use of the method of triadic comparisons. Manuscript submitted for publication. 
Levin, C. A., \& Haber, R. N. (in press). The role of visual angle as a determiner of perceived inter-object distance. Perception \& Psychophysics.

Lockman, J. J., Rieser, J. J., \& Pick, H. L., JR. (1981). Assessing blind travelers' knowledge of spatial layout. Journal of Visual Impairment \& Blindness, 7, 321-326.

loomis, J. M., Klatzky, R. L., Golledge, R. G., Cicinelli, J., Pellegrino, J., \& Fry, P. (in press). Navigation without vision: Spatial competence of blind and sighted. Journal of Experimental Psychology: General.

PrZEOREK, J. (1986). The effects of different viewing distances, viewing levels, viewing perspectives, and walking through a scene on the perception of the layout of space. Unpublished masters thesis, University of Illinois at Chicago.

Rieser, J. J., Guth, D. A., \& Hill, E. W. (1986). Sensitivity of perspective structure while walking without vision. Perception, 15, 173-188

Rieser, J. J., Hill, E. W., Talor, C. R., Bradfield, A., \& Rosen, S. (1992). Visual experience, visual field size, and the development of nonvisual sensitivity to the spatial structure of outdoor neighborhoods explored by walking. Journal of Experimental Psychology: General, 121, 210-221.

Rieser, J. J., Lockman, J. J., \& Pick, H. L., JR. (1980). The role of visual experience in knowledge of spatial layout. Perception \& Psychophysics, 28, 185-190.

Siegel, A. W., \& White, S. H. (1975). Development of spatial representations of large scale environments. In $\mathrm{H}$. W. Reese (Ed.), Advances in child development and behavior (Vol. 10, pp. 9-55). New York: Academic Press.

TOYE, R. C. (1986). The effect of viewing position on the perceived layout of space. Perception \& Psychophysics, 40, 85-92.

TVERSKY, B. (1981). Distortion in cognitive maps. Cognitive Psychology, 13, 407-433.

W AGner, M. (1985). The metric of visual space. Perception \& Psychophysics, 38, 483-495.

W ARREN, D. H. (1984). Blindness and early childhood development (2nd ed.). New York: American Foundation for the Blind.

WIEST, W. M., BELL, B. (1985). Stevens's exponent for psychophysical scaling of perceived, remembered, and inferred distance. Psychological Bulletin, 98, 457-470.

WILKINSON, L. (1986). SYSTAT: A statistical package. Evanston, IL: SYSTAT, Inc.

\section{NOTES}

1. Subjects were also asked to make relative distance estimates after they had finished estimating all of the distances in feet (or meters). How- ever, this second kind of estimation was soon abandoned in testing the blind subjects because it was very difficult for them to keep in mind which triad of objects was being judged, and the 120 triads (among 45 objects) took them well over an hour to complete. Furthermore, Levin (1992) has shown that relative distance estimation procedures are severely limited, whether analyzed directly or through MDS procedures. The limitation stems from the impossibility of recovering a monotonic ordering of true differences from the summing of ranks among the triplets of paired judgments. Consequently, even if the subject were perfect in making relative distance judgments, the resulting ordering would be less than perfect, and the scaling of those perfect judgments would produce residual stress in MDS. Levin's analysis suggests that the previous data from relative distance estimations based on the method of triads collected on blind subjects seriously underestimates their spatial knowledge. He argues that the method of triads should be abandoned.

2. The input matrix was made up of the integer values for the interobject distances given by the subject, which we were comfortable in using directly because the regression analyses indicated metrical properties of the estimates. The MDS analyses were done with ALSCAL in SAS. However, as a precaution to guard against the demands of metric scaling, we also recomputed all 34 of the MDS solutions by using a nonmetric scaling, which in effect converted each of the 45 estimates of a subject into ranks, so that the input matrix contained only ordinal values. The MDS computational program used SYSTAT (Wilkinson, 1986) and is derived from Kruskal and Wish (1978). Although the resulting stress values were uniformly lower with the nonmetric solutions, every conclusion drawn from the results based on the metric solutions was replicated in those based on nonmetric solutions, and vice versa. Only data based on the metric solutions are reported.

3. Several other methods of comparing scaled maps with true maps have been reported. As one example, Toye (1986) created a statistic based on the discrepancy, in feet, between the true coordinate location of an object and the MDS-derived location of the object, averaged over all of the objects in the scene. This mean discrepancy could then be compared across conditions, groups, and subjects. To estimate the sampling properties of this statistic, he reported the results of a Monte Carlo procedure following random matching of scaled and true locations. As a different example, Krumhansl (1979) used a program called CONGRU, which rotates and normalizes the locations in the scaled map to maximize the agreement with the locations in the true map. The present procedure (Leland Wilkinson, personal communication, 1986) is similar in principle to that underlying CONGRU, although there are some computational differences.

Unfortunately, there have been no direct comparisons among these methods to aid researchers in choosing which would be most revealing.

(Manuscript received February 20, 1992; revision accepted for publication December 31, 1992.) 